\title{
Evans Stage I
}

National Cancer Institute

\section{Source}

National Cancer Institute. Evans Stage I. NCI Thesaurus. Code C85408.

Tumor confined to the original site of growth. 